\title{
Polydopamine/silver hybrid coatings on soda-lime glass spheres with controllable release ability for inhibiting biofilm formation
}

\author{
Quanbin Shi ${ }^{1}$, Hongwei Zhang ${ }^{1 *}$, Hongping Zhang ${ }^{2,3}$, Peng Zhao ${ }^{1}$, Yuan Zhang ${ }^{1}$ and \\ Youhong Tang ${ }^{3 *}$
}

\begin{abstract}
Filtering media is anything placed in a filter that changes the quality of water flowing through it. With the variety of media available, proper selection of filter media is of great importance for filter performance. Recycled glass is evaluated as an alternative to silica sand in media filters and is an effective medium with the advantages of lower cost than silica sand, more environmental friendliness as it is a recycled product, and ease to pulverize into different sizes for specific design requirements. However, the filtration efficiency of regenerated recycled glass is limited by the formation of biofilms on its surfaces due to the large number of microorganisms such as bacteria and algae existing in the water. In this study, hydrofluoric (HF) acid etched glass spheres (GSs) modified with polydopamine (PDA) and silver nanoparticles (PDA-AgHF/GSs) were fabricated on the surface of soda-lime GSs by HF etching, crystal in situ growth, and PDA coating. HF etching and the modification of PDA coating imparted good hydrophilicity to PDA-Ag-HF/GSs. The modification of the silver coating also rendered PDA-Ag-HF/GSs excellent antibacterial properties and reduced Chlorella adhesion, and inhibited microorganism growth ability by releasing $\mathrm{Ag}^{+}$. The catechol functional group on the PDA coating could regulate the $\mathrm{Ag}^{+}$release by chelation. Good antibacterial properties, anti-algae adhesion, and controlled release of $\mathrm{Ag}^{+}$indicate that PDA-Ag-HF/GS coating can effectively inhibit the formation of biofilm on the surface of the material, providing a new strategy for the formation of anti-biofilm.
\end{abstract}

Keywords: water treatment, biofilm, antibacterial, glass sphere, multifunctionality

\section{INTRODUCTION}

Water pollution is an important problem facing human beings [1]. The presence of contaminants, such as organisms [2], heavy metal ions [3], microorganisms, and radionuclide, makes wastewater treatment procedures a complex issue involving physics, chemistry, and biology $[4,5]$. Medium filtration has been one of the most widely used methods in water treatment due to its simple operation, low cost, high efficiency, and reliability [6,7]. Among diverse filtering media, recycled glass can effectively remove particulates, organic matter, and heavy metal ions in wastewater, markedly reducing the turbidity of water $[8,9]$. However, the filtration efficiency of regenerated recycled glass can be always limited by the formation of biofilms on its surfaces due to the large amount of microorganisms such as bacteria and algae existing in the water $[10,11]$. The presence of these biofilms severely reduces the ability of the filter media and, in severe cases, increases the risk of equipment damage. Benefiting from advances in the anti-bioadhesive technology, various surfaces with biofilm inhibition capabilities supported by different surface modification methods have received extensive attention. Among them, Ag- [12], Cu- [13], and Au-based materials can effectively inhibit the bio-adhesion of microorganisms by releasing specific substances that are harmful to microorganisms [14]. Unfortunately, the release of these substances is uncontrollable and often environmentally unfriendly $[15,16]$. Therefore, controlling the release of harmful substances is of great significance for the development of

\footnotetext{
${ }^{1}$ School of Environmental Science and Engineering, Tianjin University, Tianjin 300072, China

${ }^{2}$ Engineering Research Center of Biomass Materials, Ministry of Education, Southwest University of Science and Technology, Mianyang 621010, China

${ }^{3}$ Institute for NanoScale Science and Technology, College of Science and Engineering, Flinders University, Tonsley 5042, Australia

* Corresponding authors (emails: hwzhang@tju.edu.cn (Zhang H); youhong.tang@flinders.edu.au (Tang Y))
} 
advanced water filtration media.

Dopamine (DA) has attracted much research attention as a product of biomimetic mussel adhesion chemistry [17]. Rich catechol functional groups endow DA with good adhesion, adsorption, and reduction abilities [18]. The catechol hydroxyl group can be adhered to the surface of most substrates, such as metals, ceramic, glass, and polytetrafluoroethylene by hydrogen bonding and $\pi-\pi$ stacking $[19,20]$. Meanwhile, they can adsorb harmful substances in water such as heavy metal ions [21] and organic substances [22]. More importantly, the catechol functional group has a reducing ability and can form a redox balance [23]. Such excellent performances make DA widely used in many fields such as biomedicine [24] and the environment [18].

In this study, hydrofluoric (HF) acid etched glass spheres (GSs) filter modified with polydopamine (PDA) and silver nanoparticles (PDA-Ag-HF/GSs) with controlled release and anti-biofilm ability were prepared by an acid etching and in situ growth technique, as shown in Fig. 1. Firstly, the surfaces of soda-lime GSs were etched by HF to increase the surface roughness; the contact area between the filter medium and sewage could be increased in turn. Secondly, silver ions $\left(\mathrm{Ag}^{+}\right)$were adsorbed onto the GS surfaces due to the negative charge of the surfaces, and then silver nanoparticles were grown in situ under hydrothermal conditions to construct a microenvironment with microbial toxicity. Finally, the chemical re- activity of the soda-lime GSs was improved by increasing the reactive functional groups (amino and hydroxyl groups) through the induction of PDA coatings. The abundant chemical functional groups could not only inhibit the release of $\mathrm{Ag}^{+}$, but also reduce $\mathrm{Ag}^{+}$to $\mathrm{Ag}$ nanoparticles and maintain the dissolution equilibrium of $\mathrm{Ag}^{+}$.

\section{EXPERIMENTAL SECTION}

\section{Materials}

The soda-lime GSs (composition in mol\%: C $11.06 \%$, Ca $4.51 \%$, O $51.51 \%$, Na $10.32 \%$, Si $22.60 \%$ ) used in this study were purchased from Senno Glass Beads Co., Ltd., China. Silver nitrate $\left(\mathrm{AgNO}_{3}\right)$, sodium chloride $(\mathrm{NaCl})$, and HF were purchased from Kelong Chengdu, China. DA, agar, yeast extract and peptone were purchased from Sigma, Germany. All chemicals and solvents were of reagent grade.

\section{Methods}

\section{Hydrofluoric acid etched GSs (HF/GSs)}

The soda-lime GSs were ultrasonically washed three times with ultrapure water and ethanol before use. GSs $(10 \mathrm{~g})$ were added into a reactor with polytetrafluoroethylene as the inner cover, and $20 \mathrm{~mL}$ of $20 \mathrm{wt} \% \mathrm{HF}$ was added for etching. After reacting at room temperature for $10 \mathrm{~min}$,

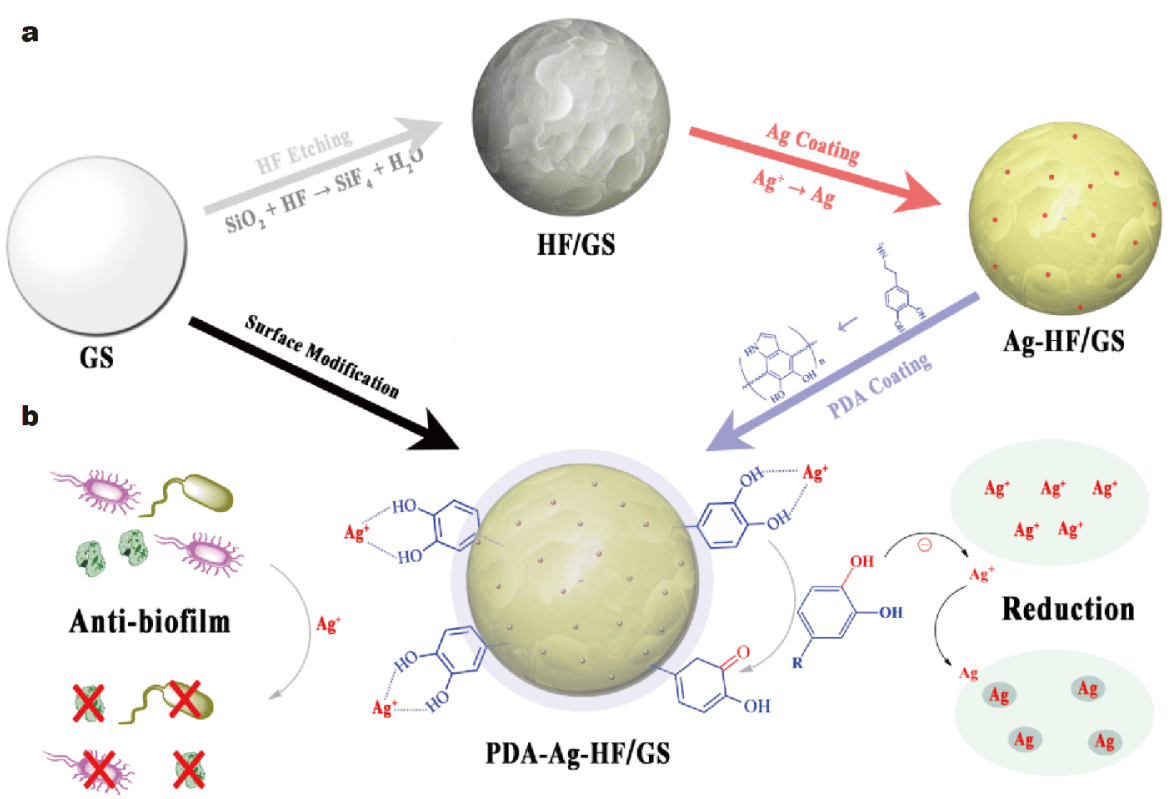

Figure 1 (a) Surface modification processes of glass sphere (GS) and (b) anti-biofilm and controlled release properties of PDA-Ag-HF/GS. 
the GSs were alternately washed with water and ethanol and finally dried at $60^{\circ} \mathrm{C}$ to obtain $\mathrm{HF} / \mathrm{GSs}$. HF could bring about a serious safety threat due to the strong causticity and toxicity. Thus, the experiments should be carried out carefully in a fuming cupboard.

Preparation of silver-modified HF/GSs

With $10 \mathrm{~g}$ of $\mathrm{HF} / \mathrm{GSs}$ in the beaker, $50 \mathrm{~mL}$ of $\mathrm{AgNO}_{3}$ solution was added to the system. Stirring was continued for $10 \mathrm{~min}$ to achieve adsorption equilibrium. Finally, the reaction system was transferred to the reactor and reacted at $180^{\circ} \mathrm{C}$ for $5 \mathrm{~h}$. The silver-modified HF/GSs (Ag-HF/ GSs) were obtained after centrifugation and washing.

Preparation of PDA-modified Ag-HF/CSs

First, $10 \mathrm{mg}$ of DA was added to $10 \mathrm{~mL}$ of $\mathrm{HCl}$-tris solution, stirred and dissolved, and then $10 \mathrm{~g}$ of Ag-HF/GSs were added. The DA was polymerized by stirring at room temperature for $12 \mathrm{~h}$. Finally, the PDA-Ag-HF/GSs were obtained by centrifugation, washing, and drying.

\section{Characterization of the spheres}

The physicochemical properties of GSs, HF/GSs, Ag-HF/ GSs and PDA-Ag-HF/GSs were characterized by scanning electron microscopy (SEM, Zeiss, Ultra55), X-ray diffraction (XRD, Panalytical X'PERT PRO) and X-ray photoelectron spectroscopy (XPS, ThermoFisher Scientific, ESCALAB 250Xi). The hydrophilic and hydrophobic properties of the materials were evaluated by testing the static water contact angles (Kruss, K100) of the surfaces.

\section{RESULTS AND DISCUSSION}

\section{Microscopic morphology of GSs}

The roughness of the GS surface can be increased by HF etching. The microstructure of a GS is shown in Fig. 2a. A smooth sphere can be observed under low magnification SEM, and some particles can be seen on the surface at high magnification SEM. After HF etching, pits about $30 \mu \mathrm{m}$ in size are clearly observed on the surface of the GS (Fig. 2b), and the pits have smooth surfaces. This is mainly because GS contains a large amount of $\mathrm{SiO}_{2}$ that can react with $\mathrm{HF}$, and after $\mathrm{SiO}_{2}$ is etched, pits will be formed on the surface of GS. The presence of pits helps to increase the specific surface area of a GS, which is beneficial to water filtration systems [25].

GSs can induce the formation of nanoparticles and PDA coatings in situ. After immersion in $\mathrm{AgNO}_{3}$ solution for hydrothermal reaction, pits of the Ag-HF/GSs were clearly observed to be coated with nanoparticles (Fig. 2c) with the diameter of $100 \mathrm{~nm}$, uniformly dispersed on the inner wall. The elemental mapping at the microstructural level by SEM with energy dispersive X-ray spectrometry (EDS) (Fig. 2e) also shows that the silver distribution in the nanoparticles is uniform. This may be due to the fact that HF etches GSs, causing a large number of defects on the surfaces. Those defects facilitate the nucleation of nanoparticles and ultimately induce the growth and formation of nanoparticles. After modification by PDA coating, most of nanoparticles on the surface of PDA-AgHF/GSs (Fig. 2d) are embedded in the coating and the
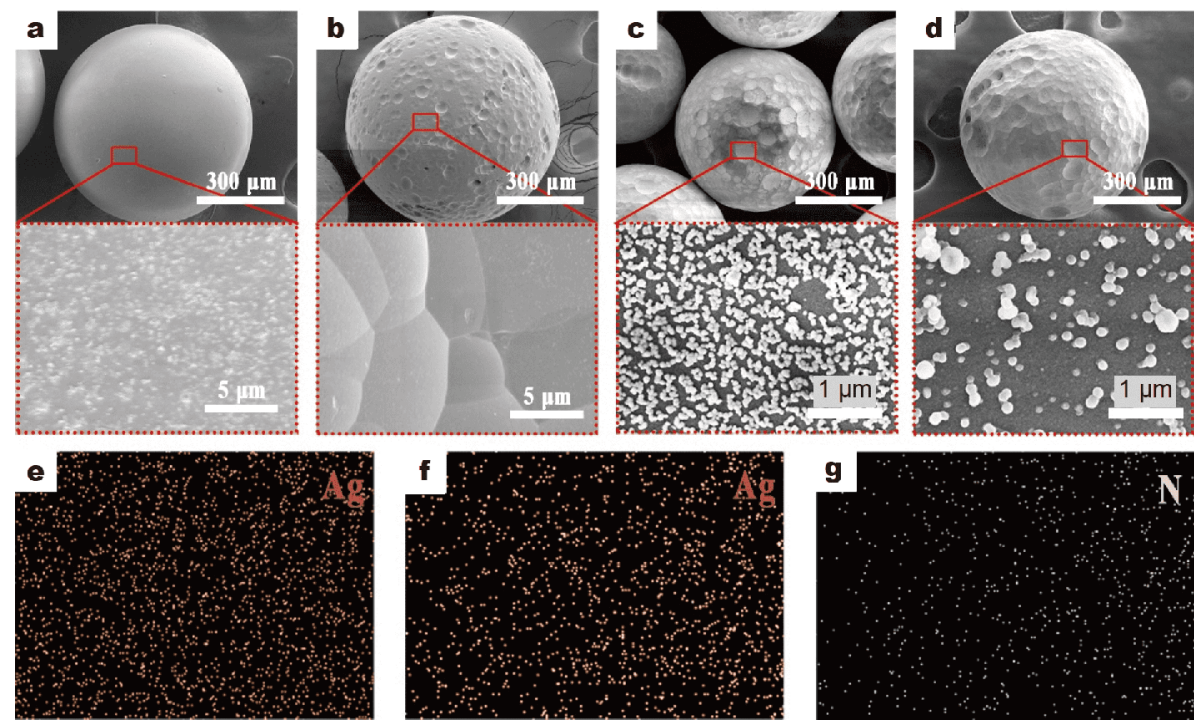

Figure 2 The microstructure of GSs. (a-d) are SEM images of GS, HF/GS, Ag-HF/GS and PDA-Ag-HF/GS, respectively. (e) and (f) are Ag element mapping of Ag-HF/GS and PDA-Ag-HF/GS, and (g) is N element mapping of PDA-Ag-HF/GS. 
particle size increases. DA has catechol functional groups that can be adsorbed on the surfaces of GSs and nanoparticles by hydrogen bonding and polymerized under alkaline conditions to form a coating of PDA. This explains the inlay of the nanoparticles and the increase in the size of surface particles. The elemental mapping also shows a uniform distribution of Ag (Fig. 2f) and N (Fig. 2g) elements on the surface of a GS, indicating successful modification of the PDA coating.

\section{Chemical structure of the surface of GSs}

Surface analysis results show that Ag nanoparticles and PDA coatings are successfully modified on the surface of the GSs. No significant diffraction peaks on the XRD pattern (Fig. 3a) can be observed, indicating that the GS is in an amorphous state. The modification process of etching and the later functional coating do not cause any change in the GS. Moreover, the survey XPS spectrum of a GS is shown in Fig. 3b, where the GS and HF/GS clearly exhibit peaks at 105,285 and $534 \mathrm{eV}$ that belong to $\mathrm{Si} 2 \mathrm{p}$,
C 1s, and O 1s, respectively. For both Ag-HF/GS and PDA-Ag-HF/GS, a peak of Ag is observed around $375 \mathrm{eV}$, and the $\mathrm{N} 1 \mathrm{~s}$ peak $(401 \mathrm{eV})$ is also observed on the PDAAg-HF/GS.

By fitting the high-resolution spectrum (Fig. $3 \mathrm{c}$ and d) before and after etching, it is found that O 1s on GSs can be fitted to two peaks of 532.48 and $530.86 \mathrm{eV}$ which correspond to the $\mathrm{Si}-\mathrm{O}$ bond in the glass and the hydroxyl $(-\mathrm{OH})$ adsorbed on the surface. However, after the HF etching, the proportion of hydroxyl oxygen $(-\mathrm{OH})$ increases from $8.4 \%$ to $15.5 \%$ (Table 1), indicating that more defects have been added onto the surface of the GSs after etching, and the defects cause an increase in the content of hydroxyl oxygen $(-\mathrm{OH})$. High-resolution spectrum of Ag 3d (Fig. 3e) can be fitted to two peaks of 368.24 and $374.25 \mathrm{eV}$ that correspond to $\mathrm{Ag} 3 \mathrm{~d}_{5 / 2}$ and $\mathrm{Ag}$ $3 \mathrm{~d}_{3 / 2}$, respectively $[26,27]$. This finding indicates that the nanoparticles on the surface are $\mathrm{Ag}$ nanoparticles. It is worth noting that, because the Ag nanoparticle content is too low, almost no characteristic diffraction peak of $\mathrm{Ag}$
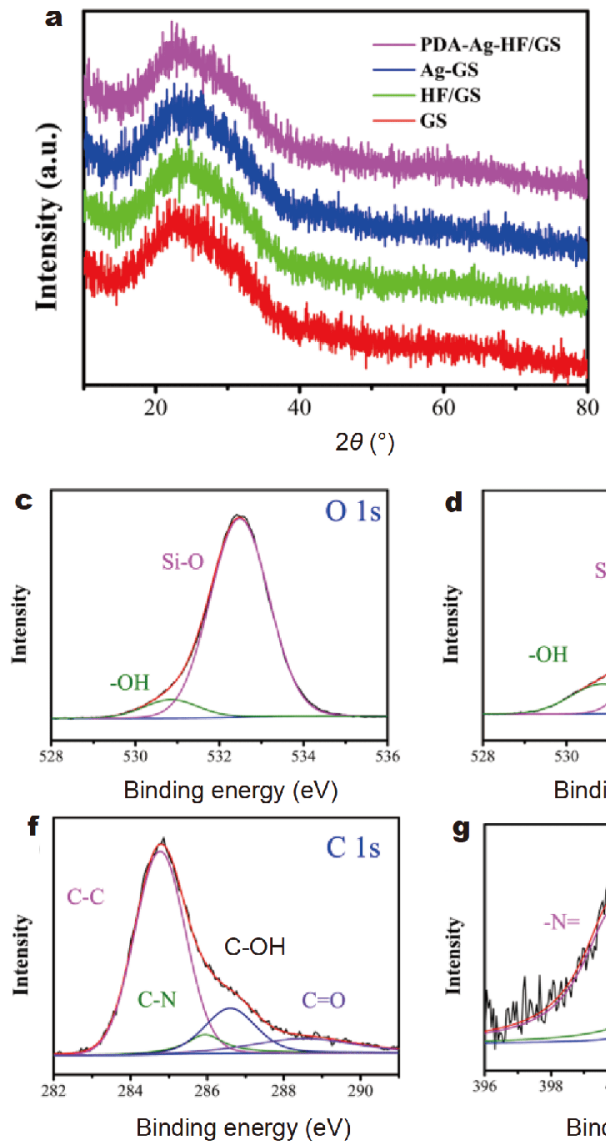
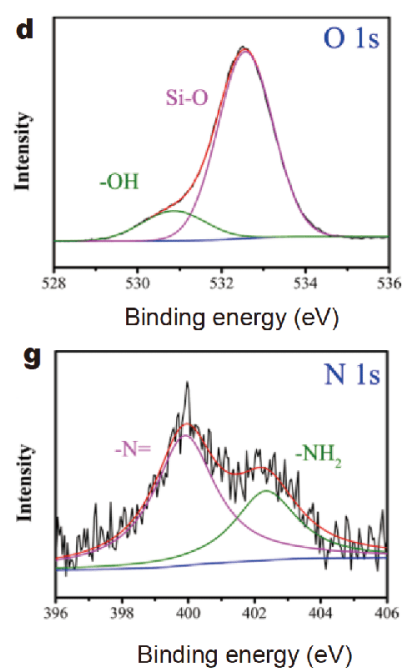
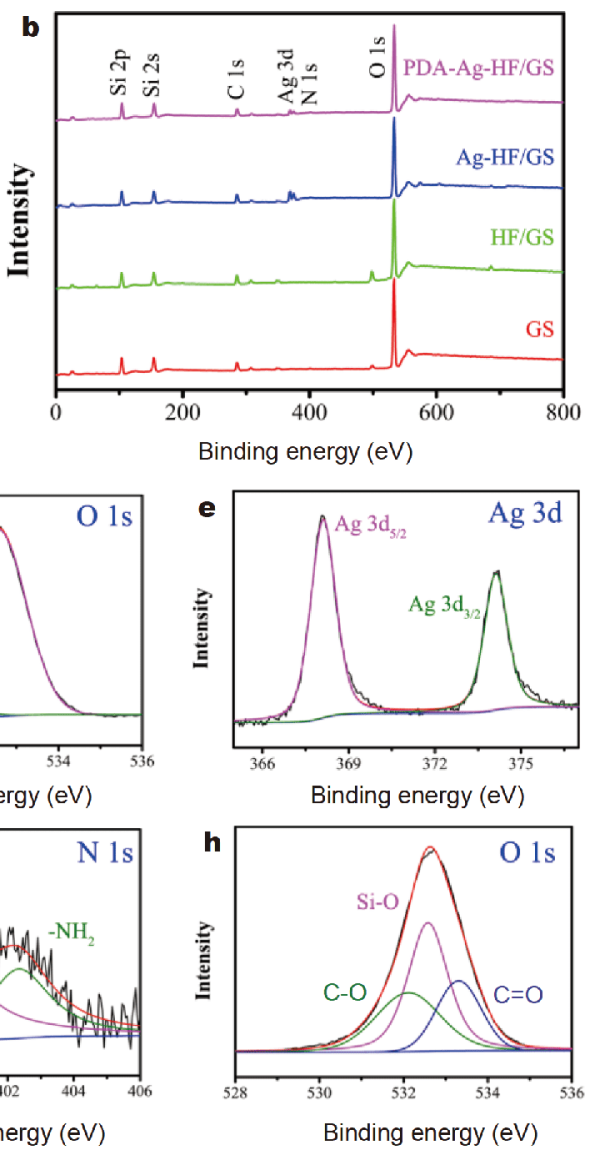

Figure 3 Chemical compositions of the GS surface. (a) XRD spectra of GSs. (b) XPS of GSs. High-resolution O 1s spectra of (c) GSs and (d) HF/GSs. (e) High-resolution Ag 3d spectra of Ag-HF/GSs. (f-h) are the high-resolution C 1s, N 1s, and O 1s spectra of PDA-Ag-HF/GSs, respectively. 
Table 1 Curve fitting results for the O 1s spectra of GSs and HF/GSs

\begin{tabular}{cccc}
\hline Function group & $\begin{array}{c}\text { Binding energy } \\
(\mathrm{eV})\end{array}$ & GS (\%) & HF/GS (\%) \\
\hline$-\mathrm{OH}$ & 530.85 & 8.4 & 15.5 \\
$\mathrm{O}-\mathrm{Si}$ & 532.48 & 91.6 & 84.5 \\
\hline
\end{tabular}

can be observed on the XRD pattern. Finally, the fitting results of $\mathrm{C} 1 \mathrm{~s}, \mathrm{~N} 1 \mathrm{~s}$, and $\mathrm{O} 1 \mathrm{~s}$ of PDA-Ag-HF/GSs (Fig. 3f-h) show that the $\mathrm{C} 1 \mathrm{~s}$ spectrum can be fitted to four peaks at $284.77,285.94,286.6$ and $288.52 \mathrm{eV}$, corresponding to the carbon skeleton $(\mathrm{C}-\mathrm{C})$, amino group $(\mathrm{C}-\mathrm{N})$, phenolic hydroxyl group $(\mathrm{C}-\mathrm{OH})$, and carbonyl group $(\mathrm{C}=\mathrm{O})$ on $\mathrm{PDA}$, respectively [24]. The peaks at 399.90 and $402.34 \mathrm{eV}$ on the $\mathrm{N} \mathrm{1s}$ spectrum represent amide and the amino groups $\left(-\mathrm{NH}_{2}\right)$, respectively. The presence of $\mathrm{C}-\mathrm{O}$ and $\mathrm{C}=\mathrm{O}$ can also be observed at 531.1 and $533.31 \mathrm{eV}$ on the $\mathrm{O} 1 \mathrm{~s}$ spectrum, indicating that $\mathrm{DA}$ has polymerized on the surface and formed a PDA coating.

\section{Wettability of the GS surface}

The wettability test results show that PDA-Ag-HF/GSs have good surface wettability. As shown in Fig. 4a, when the droplets were dropped onto the surface of GSs, they were semi-circular on the surface of the glass sheet, with a water contact angle of $60^{\circ}$. When the surface was etched by HF, the droplets began to spread, and the water contact angle dropped to $41^{\circ}$. The droplets were completely spread on the Ag-HF/GS surface after the silver nanoparticles were loaded, with a contact angle of $13^{\circ}$. Interestingly, the spread was finally reduced on the PDA-Ag$\mathrm{HF} / \mathrm{GS}$ and the contact angle increased to $31^{\circ}$. This is mainly due to the fact that the modification of PDA coating reduces the roughness of the surface. The silver nanoparticles are evenly distributed on the surface, which increases the surface roughness of GS. Other research results also demonstrated that the spread of water can be promoted with the improved roughness [28,29]. However, with the modification of PDA coating, the silver nanoparticles were embedded in the PDA coating. The relative flat PDA coating can reduce the roughness to some extent and lead to a decrement in wettability.

\section{Silver release behavior of GSs}

The cumulative release curve of $\mathrm{Ag}^{+}$was used to investigate the release behavior of the coating for $\mathrm{Ag}^{+}$[30]. GSs $(1 \mathrm{~g})$ were immersed in $80 \mathrm{~mL}$ of deionized water. The solution was taken out every $24 \mathrm{~h}$ for measuring the cumulative release concentration in it by atomic absorption spectroscopy (AAS, PerkinElmer, AA700). The cumulative release of $\mathrm{Ag}^{+}$showed that the modification of the PDA coating helped to inhibit the release of $\mathrm{Ag}^{+}$on Ag-HF/GSs. As shown by the ion release curve (Fig. 5), $\mathrm{Ag}$ ions had a rapid release rate in the first 4 days in AgHF/GSs without PDA coating, and the release rate decreased after 4 days, with the release concentration of 55 ppb at 5 days. With modification by the PDA coating, the release rate of $\mathrm{Ag}^{+}$on PDA-Ag-HF/GSs decreased in the first 4 days, and the release concentration after 4 days was $23 \mathrm{ppb}$ lower than that of the Ag-HF/GSs. This was mainly due to the fixation of $\mathrm{Ag}^{+}$by the PDA coating on the GS surface. The PDA surface was rich in catechol functional groups that could adsorb $\mathrm{Ag}^{+}$and reduce it to elemental Ag [31], resulting in the decrease in the release of $\mathrm{Ag}^{+}$.

\section{Adhesion of Chlorella}

Chlorella adhesion experiments were used to explore the anti-algae adhesion of GSs [32]. The GSs were placed in a suspension of Chlorella $\left(8 \times 10^{6}\right.$ cells $\left.\mathrm{mL}^{-1}\right)$ and after $24 \mathrm{~h}$ of culture were fixed with glutaraldehyde (2 wt.\%) and freeze-dried. Finally, Chlorella on the surface of the GSs

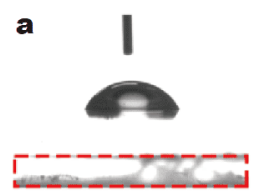

GS

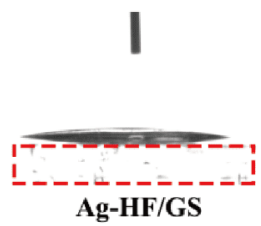

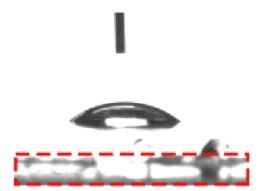

HF/GS

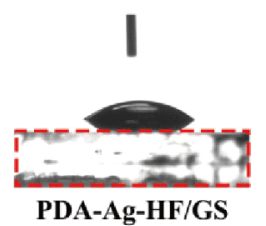

b

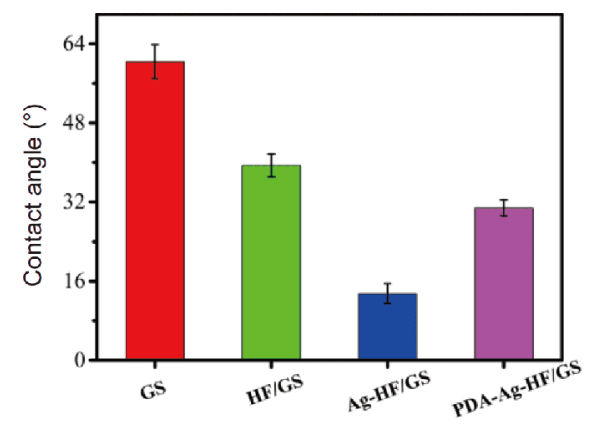

Figure 4 (a) Photo images and (b) water contact angle for $5 \mu \mathrm{L}$ droplet on the surfaces of GS, HF/GS, Ag-HF/GS and PDA-Ag-HF/GS, respectively. 


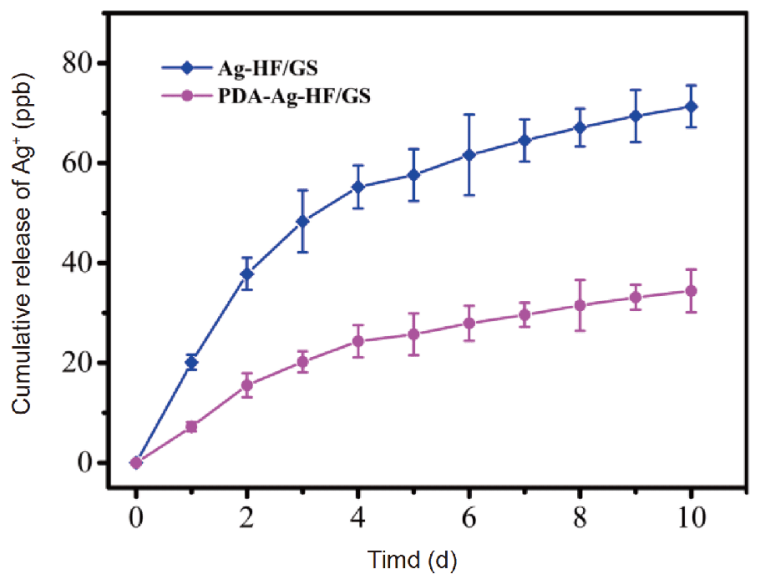

Figure 5 Cumulative release of $\mathrm{Ag}^{+}$.

was observed by SEM. SEM images of Chlorella adhesion indicated that the silver-modified surface effectively inhibited Chlorella adhesion. As shown in Fig. 6, a large amount of Chlorella adhesion was observed on the surfaces of GSs and HF/GSs and a dense Chlorella membrane was formed. However, on the surface of the Agmodified Ag-HF/GSs, almost no Chlorella adhered to the surface. Moreover, Chlorella adhered to the surface of PDA-Ag-HF/GSs, but the growth of the Chlorella was inhibited. This was mainly due to the ability of Chlorella to generate adhesion proteins that adhered to the surface of GSs $[33,34]$. However, much $\mathrm{Ag}^{+}$was released on the surface of the Ag-modified surface and $\mathrm{Ag}^{+}$could be bound to the surface to cause changes in the cell membrane [35]. Some studies have reported that the entry of $\mathrm{Ag}^{+}$into cells could upregulate the concentration of intracellular reactive oxygen species, ultimately leading to cell apoptosis [36]. Finally, due to the abundant func-
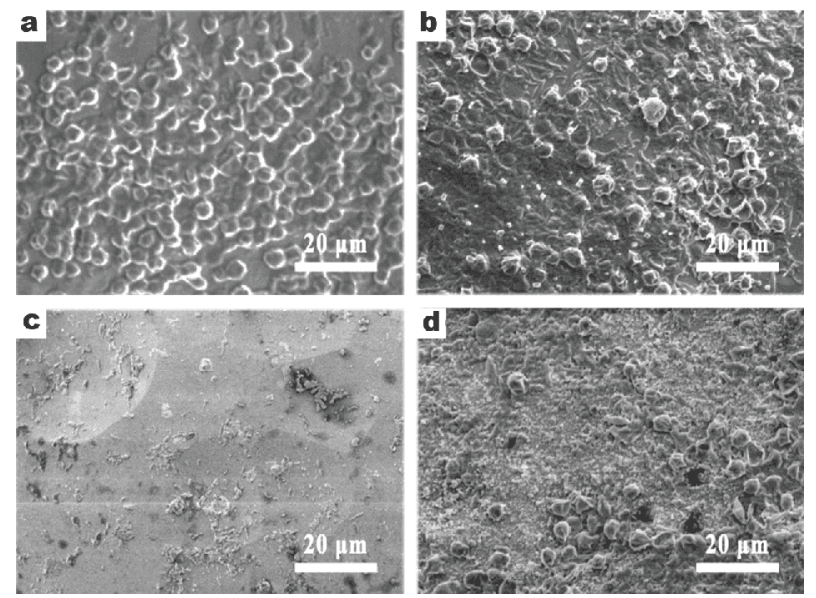

Figure 6 Adhesion of Chlorella on the surfaces of (a) GS, (b) HF/GS, (c) Ag-HF/GS, and (d) PDA-Ag-HF/GS. tional groups on PDA, the adhesion of Chlorella could be promoted, but the released $\mathrm{Ag}^{+}$also strongly affected physiological metabolism, eventually leading to the apoptosis of Chlorella on the surface.

\section{Adhesion of bacteria}

The antibacterial adhesion and bacteriostatic ability of GSs were investigated by Escherichia coli (E. coli) and Bacillus. After co-cultivation of GSs and bacteria, SEM and an absorbance experiment were used to detect the bacterial adhesion and growth [37]. The results show that the silver nanoparticle coating surface can effectively inhibit bacterial adhesion. The adhesion behavior of $E$. coli on the GS surface is shown in Fig. 7a-d. A large amount of $E$. coli adhesion can be observed aggregated on the surfaces of GSs and HF/GSs, as indicated by the red arrows. In contrast, only a small number of bacteria adhere to the Ag-HF/GSs surface and there is almost no adhesion of bacteria on the PDA-Ag-HF/GSs surface. Similar to $E$. coli, adhesion of Bacillus is observed on the surface of GSs and HF/GSs without $\mathrm{Ag}$ coating modification, as indicated by the blue arrows in Fig. $7 \mathrm{e}-\mathrm{h}$, whereas on the surfaces of Ag-HF/GSs and PDA-Ag-HF/GSs modified by Ag coating, such coating is not observed. This is mainly due to the fact that the released $\mathrm{Ag}^{+}$from the silver coating can enter bacteria and interfere with the normal physiological activities of bacteria. Studies have shown that $\mathrm{Ag}^{+}$can bind to bacterial cell membranes, altering the charge properties on the surface of cell membranes and disrupting the structure of cell membranes, inhibiting the formation of cell membranes during cell proliferation $[38,39]$. Moreover, $\mathrm{Ag}^{+}$can cause the cell surface ionic membrane to lose its ability to transport and transfer energy and matter, eventually killing the cells $[40,41]$.

Bacterial experiments show that PDA-Ag-HF/GSs also have excellent spectral antibacterial growth ability, as well as inhibiting bacterial adhesion. The culture media after co-cultivation of GSs and bacteria are shown in Fig. $7 \mathrm{i}$ and $\mathrm{j}$. The bacterial culture medium in the blank, GS and HF/GS groups is turbid, indicating high bacterial concentration. In contrast, the color of the medium in the Ag-coated Ag-HF/GS and PDA-Ag-HF/GS groups is clear, indicating low bacterial concentration. Quantitative analysis by absorbance showed that the antibacterial rates of Ag-HF/GSs and PDA-Ag-HF/GSs for E.coli (Fig. 7k) were $86 \%$ and $82 \%$, and the antibacterial rates of Bacillus (Fig. $7 \mathrm{l}$ ) were $87 \%$ and $84 \%$. The good antibacterial ability was mainly due to the release of $\mathrm{Ag}^{+}$from the surfacemodified Ag layer, which had spectral antibacterial properties to kill bacteria in the environment [38]. 

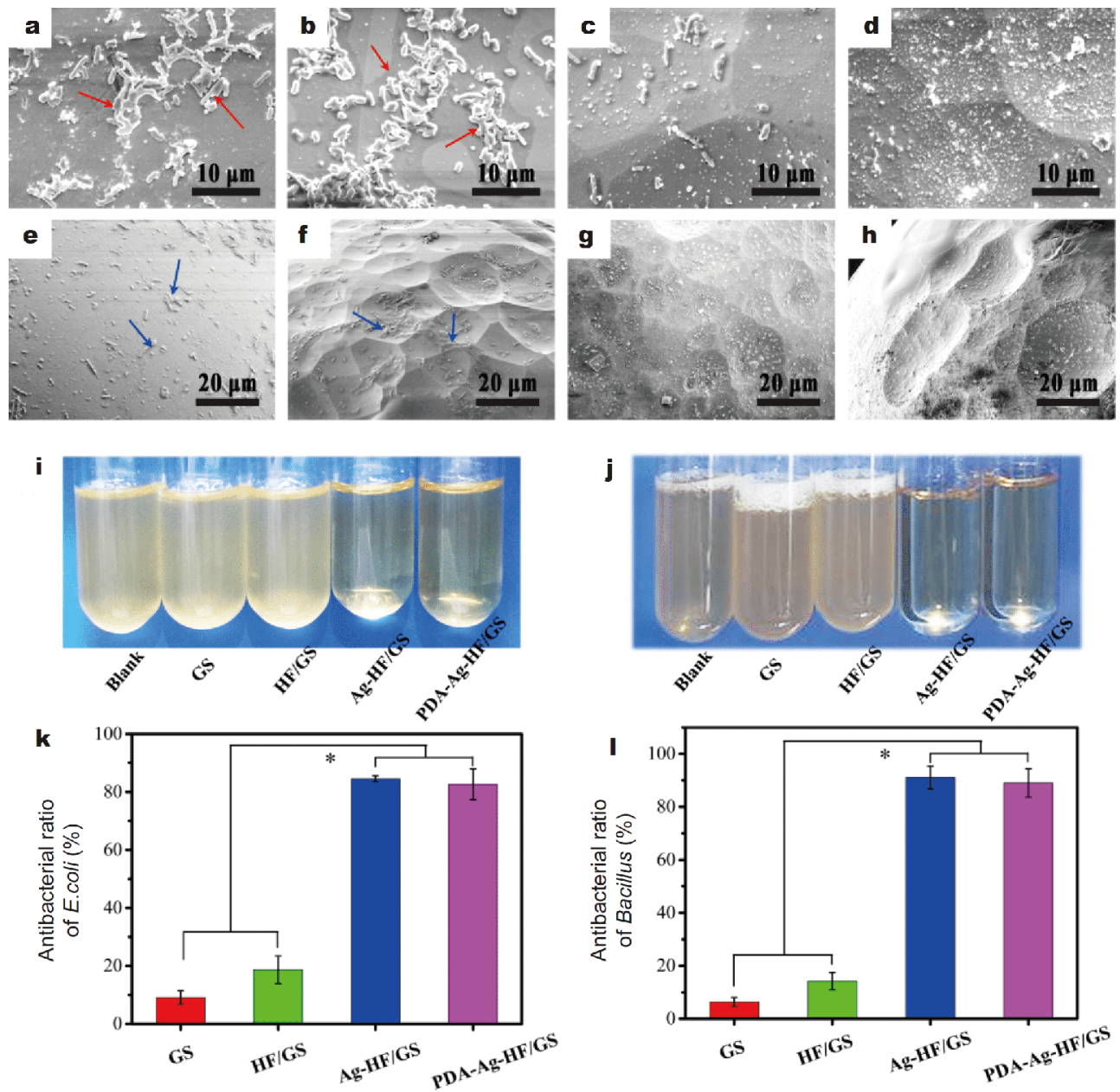

Figure 7 Adhesion of bacteria on the surface of GSs. SEM images of E. coli adhesion on the surfaces of (a) GSs, (b) HF/GSs, (c) Ag-HF/GSs and (d) PDA-Ag-HF/GSs. SEM images of Bacillus adhesion on the surfaces of (e) GSs, (f) HF/GSs, (g) Ag-HF/GSs and (h) PDA-Ag-HF/GSs. (i) and (j) are photographs of different GSs co-cultured with E. coli and Bacillus. (k) and (l) are the antibacterial ratios of different GSs against E. coli and Bacillus.

Although PDA-Ag-HF/GS owns good water wettability and the ability to inhibit microbial growth, there are still some limitations, such as relatively high cost, treatment of by-products, and dynamic antibacterial ability that need to be further optimized. Firstly, approximately $0.025 \mathrm{~g}$ of silver nitrate was needed to produce $10 \mathrm{~g}$ of PDA-Ag-HF/ GS glass beads. Reducing the usage of silver nitrate will be helpful to reduce the cost of filter media. Secondly, the treatment of by-products should also be considered. During HF etching process, silicon fluoride $\left(\mathrm{SiF}_{4}\right)$ gas, precipitation of $\mathrm{CaF}_{2}$ and silicic acid would be generated. Producing $10 \mathrm{~g}$ of PDA-Ag-HF/GS will generate about $20 \mathrm{~mL}$ wastewater with the main component of $\mathrm{NaF}$. Among them, $\mathrm{CaF}_{2}$ and silicic acid can be separated by precipitation, and $\mathrm{NaF}$ can also be separated into lowerlevel industrial raw materials. Finally, for the dynamic antibacterial ability, the filtration process is complex involving the flow of microbial population, the flow rate of filtration solution, and the flow rate of solution. These factors will intensely affect the release of silver ions and ultimately affect the ability to inhibit microbial growth. Therefore, the best filtration procedure should be selected according to the pollutants, such as bacterial species and chemical composition in different water qualities. The ion release and long-term antimicrobial growth ability of the filter medium can finally be evaluated.

\section{CONCLUSION}

In this study, multifunctional PDA-Ag-HF/GSs were prepared on the surface of soda-lime GSs by HF etching, crystal in situ growth, and PDA coating modification. Among them, HF etching and the modification of PDA 
coating imparted good hydrophilicity to PDA-Ag-HF/ GSs. Moreover, the modification of the silver coating also gave PDA-Ag-HF/GSs excellent antibacterial properties and Chlorella adhesion, but inhibited microorganism growth ability by releasing $\mathrm{Ag}^{+}$. The catechol functional group on the PDA coating could regulate the $\mathrm{Ag}^{+}$release by chelation. Good antibacterial properties, anti-algae adhesion, and controlled release of $\mathrm{Ag}^{+}$indicate that PDA-Ag-HF/GS coating can effectively inhibit the formation of biofilm on the surface of the material, providing a new strategy for the formation of anti-biofilm.

Received 15 November 2019; accepted 13 January 2020; published online 4 March 2020

1 Adeleye AS, Conway JR, Garner K, et al. Engineered nanomaterials for water treatment and remediation: Costs, benefits, and applicability. Chem Eng J, 2016, 286: 640-662

2 Bolto B, Gregory J. Organic polyelectrolytes in water treatment. Water Res, 2007, 41: 2301-2324

3 Srivastava NK, Majumder CB. Novel biofiltration methods for the treatment of heavy metals from industrial wastewater. J Hazard Mater, 2008, 151: 1-8

4 Herrmann S, De Matteis L, de la Fuente JM, et al. Removal of multiple contaminants from water by polyoxometalate supported ionic liquid phases (POM-SILPs). Angew Chem Int Ed, 2017, 56: 1667-1670

5 Di Cesare A, Eckert EM, D'Urso S, et al. Co-occurrence of integrase 1 , antibiotic and heavy metal resistance genes in municipal wastewater treatment plants. Water Res, 2016, 94: 208-214

6 Huang X, Jiao T, Liu Q, et al. Hierarchical electrospun nanofibers treated by solvent vapor annealing as air filtration mat for highefficiency PM2.5 capture. Sci China Mater, 2019, 62: 423-436

7 Aslan S, Cakici H. Biological denitrification of drinking water in a slow sand filter. J Hazard Mater, 2007, 148: 253-258

8 Lim HS, Lim W, Hu JY, et al. Comparison of filter media materials for heavy metal removal from urban stormwater runoff using biofiltration systems. J Environ Manage, 2015, 147: 24-33

9 Cheng Y, Huang T, Cheng L, et al. Structural characteristic and ammonium and manganese catalytic activity of two types of filter media in groundwater treatment. J Environ Sci, 2018, 72: 89-97

10 Freitas de Oliveira F, Schneider RP. Slow sand filtration for biofouling reduction in seawater desalination by reverse osmosis. Water Res, 2019, 155: 474-486

11 Bar-Zeev E, Passow U, Romero-Vargas Castrillón S, et al. Transparent exopolymer particles: From aquatic environments and engineered systems to membrane biofouling. Environ Sci Technol, 2015, 49: 691-707

12 Mansouri J, Charlton T, Chen V, et al. Biofouling performance of silver-based PES ultrafiltration membranes. Desalin Water Treat, 2016, 57: 28100-28114

13 Park JA, Lee SC, Kim SB. Synthesis of dual-functionalized poly (vinyl alcohol)/poly(acrylic acid) electrospun nanofibers with enzyme and copper ion for enhancing anti-biofouling activities. J Mater Sci, 2019, 54: 9969-9982

14 Yin J, Deng B. Polymer-matrix nanocomposite membranes for water treatment. J Membrane Sci, 2015, 479: 256-275

15 Souza LRR, da Silva VS, Franchi LP, et al. Toxic and beneficial potential of silver nanoparticles: The two sides of the same coin. Adv Exp Med Biol, 2018, 1048: 251-262

16 Baruah S, Najam Khan M, Dutta J. Perspectives and applications of nanotechnology in water treatment. Environ Chem Lett, 2016, 14: $1-14$

17 Lee H, Dellatore SM, Miller WM, et al. Mussel-inspired surface chemistry for multifunctional coatings. Science, 2007, 318: 426430

18 Liu Y, Ai K, Lu L. Polydopamine and its derivative materials: Synthesis and promising applications in energy, environmental, and biomedical fields. Chem Rev, 2014, 114: 5057-5115

19 Ryu JH, Messersmith PB, Lee H. Polydopamine surface chemistry: A decade of discovery. ACS Appl Mater Interfaces, 2018, 10: 75237540

20 Lee HA, Ma Y, Zhou F, et al. Material-independent surface chemistry beyond polydopamine coating. Acc Chem Res, 2019, 52: 704-713

21 Hebbar RS, Isloor AM, Ananda K, et al. Fabrication of polydopamine functionalized halloysite nanotube/polyetherimide membranes for heavy metal removal. J Mater Chem A, 2016, 4: 764-774

$22 \mathrm{Fu}$ J, Chen Z, Wang M, et al. Adsorption of methylene blue by a high-efficiency adsorbent (polydopamine microspheres): Kinetics, isotherm, thermodynamics and mechanism analysis. Chem Eng J, 2015, 259: 53-61

$23 \mathrm{Lu} \mathrm{Z}$, Xiao J, Wang Y, et al. In situ synthesis of silver nanoparticles uniformly distributed on polydopamine-coated silk fibers for antibacterial application. J Colloid Interface Sci, 2015, 452: 8-14

24 Tang P, Han L, Li P, et al. Mussel-inspired electroactive and antioxidative scaffolds with incorporation of polydopamine-reduced graphene oxide for enhancing skin wound healing. ACS Appl Mater Interfaces, 2019, 11: 7703-7714

25 LaMarche CQ, Leadley S, Liu P, et al. Method of quantifying surface roughness for accurate adhesive force predictions. Chem Eng Sci, 2017, 158: 140-153

26 Ge L, Han C, Liu J, et al. Enhanced visible light photocatalytic activity of novel polymeric g- $\mathrm{C}_{3} \mathrm{~N}_{4}$ loaded with Ag nanoparticles. Appl Catal A-General, 2011, 409: 215-222

27 Zhang P, Shao C, Zhang Z, et al. In situ assembly of well-dispersed Ag nanoparticles (AgNPs) on electrospun carbon nanofibers (CNFs) for catalytic reduction of 4-nitrophenol. Nanoscale, 2011, 3: $3357-3363$

28 Spori DM, Drobek T, Zürcher S, et al. Beyond the lotus effect: Roughness influences on wetting over a wide surface-energy range. Langmuir, 2008, 24: 5411-5417

29 Drelich J, Chibowski E. Superhydrophilic and superwetting surfaces: Definition and mechanisms of control. Langmuir, 2010, 26: 18621-18623

30 Guldiren D, Aydin S. Antimicrobial property of silver, silver-zinc and silver-copper incorporated soda lime glass prepared by ion exchange. Mater Sci Eng-C, 2017, 78: 826-832

31 Ma Y, Niu H, Zhang X, et al. Colorimetric detection of copper ions in tap water during the synthesis of silver/dopamine nanoparticles. Chem Commun, 2011, 47: 12643-12645

32 Ma S, Ye Q, Pei X, et al. Antifouling on Gecko's feet inspired fibrillar surfaces: Evolving from land to marine and from liquid repellency to algae resistance. Adv Mater Interfaces, 2015, 2: 1500257

33 Sirmerova M, Prochazkova G, Siristova L, et al. Adhesion of Chlorella vulgaris to solid surfaces, as mediated by physicochemical 
interactions. J Appl Phycol, 2013, 25: 1687-1695

34 Sekar R, Venugopalan VP, Satpathy KK, et al. Laboratory studies on adhesion of microalgae to hard substrates. Hydrobiologia, 2004, 512: $109-116$

35 Zhou $\mathrm{K}, \mathrm{Hu} \mathrm{Y}$, Zhang $\mathrm{L}$, et al. The role of exopolymeric substances in the bioaccumulation and toxicity of Ag nanoparticles to algae. Sci Rep, 2016, 6: 32998

36 Hazeem LJ, Kuku G, Dewailly E, et al. Toxicity effect of silver nanoparticles on photosynthetic pigment content, growth, ROS production and ultrastructural changes of microalgae Chlorella vulgaris. Nanomaterials, 2019, 9: 914

37 Xie C, Lu X, Han L, et al. Biomimetic mineralized hierarchical graphene oxide/chitosan scaffolds with adsorbability for immobilization of nanoparticles for biomedical applications. ACS Appl Mater Interfaces, 2016, 8: 1707-1717

38 Rizzello L, Pompa PP. Nanosilver-based antibacterial drugs and devices: Mechanisms, methodological drawbacks, and guidelines. Chem Soc Rev, 2014, 43: 1501-1518

39 Mirzajani F, Ghassempour A, Aliahmadi A, et al. Antibacterial effect of silver nanoparticles on Staphylococcus aureus. Res MicroBiol, 2011, 162: 542-549

40 Li WR, Xie XB, Shi QS, et al. Antibacterial activity and mechanism of silver nanoparticles on Escherichia coli. Appl Microbiol Biotechnol, 2010, 85: 1115-1122

41 Le Ouay B, Stellacci F. Antibacterial activity of silver nanoparticles: A surface science insight. Nano Today, 2015, 10: 339-354

Acknowledgements Zhang $\mathrm{H}$ and Tang $\mathrm{Y}$ are grateful to the Longshan Academic Talent Research Supporting Program of SWUST (18LZX447) and the biofilm research \& innovation consortium from the College of Science and Engineering, Flinders University for supporting this research, respectively.

Author contributions Zhang HW and Tang Y designed the research. Shi Q fabricated the materials, did the characterizations, analyzed the results and drafted the manuscript with support from Zhang HP, Zhao P and Zhang Y. Zhang HP and Tang Y revised the manuscript. All authors contributed to the general discussion.

Conflict of interest The authors declare that they have no conflict of interest.

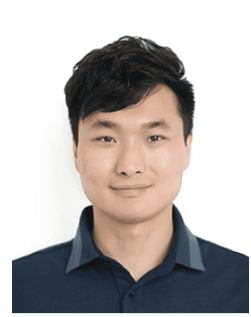

Quanbin Shi received his master's degree from Southwest University of Science and Technology in 2013. During this period, he mainly studied the degradation characteristics of degradable mulch under natural composting conditions. $\mathrm{He}$ entered the School of Environmental Science and Engineering, Tianjin University for $\mathrm{PhD}$ study in 2013. During his $\mathrm{PhD}$, his main research direction is the preparation of surface modified glass spheres and the study of their adsorption properties.

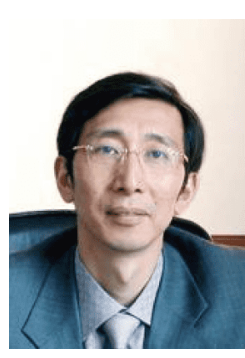

Hongwei Zhang is the Director of the Institute of Sustainable Development of Resources, Environment, Ecology and Society of Tianjin University. He graduated from Tianjin University with a major in water supply and drainage in 1982 and obtained a doctorate degree in engineering in 2002 in Tianjin University. For many years, he has been engaged in scientific research and teaching in the application of membrane technology in water treatment processes, mathematical simulation and optimal operation of urban water supply systems.

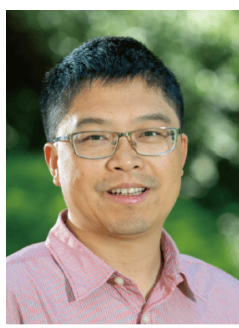

Youhong Tang obtained his $\mathrm{PhD}$ degree in the Hong Kong University of Science and Technology in 2007. He moved to Flinders University with an ARC-DECRA in 2012 from the Centre for Advanced Materials Technology, the University of Sydney. Prof. Tang is a material science and engineering researcher with research interests mainly focused on the structure-processproperty relations of polymeric materials and nanocomposites, especially on multifunctional and value-added nanocomposites and bioresources, biomaterials and biosensors, especially incorporating novel aggregation-induced emission materials.

\section{具有可控释放能力和可抑制生物膜形成的聚多巴 胺/银杂化涂层的钠钙玻璃球}

史全滨 ${ }^{1}$, 张宏伟 ${ }^{{ }^{*}}$, 张红平 ${ }^{2,3}$, 赵鹏 ${ }^{1}$, 张媛 ${ }^{1}$, 唐友宏 ${ }^{3^{*}}$

摘要 过滤介质作为过滤装置中的关键材料可改变流经其中的水 的质量. 正确选择过滤介质对于过滤装置的过滤性能至关重要. 再 生玻璃作为介质过滤装置中硅砂的替代品, 具有比硅砂廉价、环 境友好、可再生等优点, 并且可以根据特定的设计要求将其粉碎 成不同的尺寸. 然而, 水中存在大量的诸如细菌和藻类的微生物, 故再生的可循环玻璃介质的过滤效率受到在其表面富集的生物膜 的极大限制. 本研究中, 我们通过在钠钙玻璃球 (GS)表面上进行氢 氟酸 $(\mathrm{HF})$ 蚀刻和原位结晶制备了用聚多巴胺 (PDA)和银 $(\mathrm{Ag})$ 纳米 颗粒改性的氢氟酸蚀刻玻璃球(PDA-Ag-HF/GSs). 银晶体的原位生 长、HF蚀刻和PDA涂层的改性赋予了 PDA-Ag-HF/GS良好的亲水 性. 银涂层的改性还使得PDA-Ag-HF/GS具有出色的抗菌性能和较 小的球藻附着力, 且通过释放 $\mathrm{Ag}$ 离子可抑制微生物的生长. PDA涂 层上的邻苯二酚官能团可通过鳌合作用调节 $\mathrm{Ag}$ 离子的释放. 良好 的抗菌性能、抗藻类附着力和 $\mathrm{Ag}$ 离子的受控释放表明, PDA-AgHF/GS涂层可有效抑制材料表面生物膜的形成, 为抗生物膜的形 成提供了新的策略. 